\title{
Cuantificación porcentual de los daños existentes en los revestimientos interiores de las viviendas españolas
}

\section{Quantification of the percentage of existing damage in internal coatings of Spanish houses}

\author{
M. J. Carretero-Ayuso $^{(*)}$, A. Moreno-Cansado ${ }^{(*)}$
}

\section{RESUMEN}

Los revestimientos interiores son de las unidades constructivas que más interrelación tienen con los usuarios, dada su cercanía de uso con éstos. Esta investigación se centra en la determinación de los daños más usuales en los alicatados, enfoscados, guarnecidos, falsos techos y solados interiores, obtenidos en el conjunto de España a partir de los requerimientos presentados por los usuarios. Se determinan también cuáles son los orígenes que motivan estas deficiencias, así como la presencia que tienen las mismas en función del formato constructivo en donde tienen lugar (viviendas en bloque o unifamiliares). A partir de los datos manejados se presentan los grupos de daños más característicos, así como un procedimiento para determinar la probabilidad ante futuros requerimientos, en función de una matriz ponderada que clasifica la correspondencia entre los 4 descriptores manejados. El conocimiento de los resultados obtenidos ayudará a los técnicos a minimizar los daños en estas unidades constructivas.

Palabras clave: Patologías de construcción, enfoscados y guarnecidos, solados y alicatados, deficiencias.

\section{ABSTRACT}

Internal coatings are among those construction units which experience the most interaction with users, given their proximity to them. This research focuses on determining the most usual damages in tiling, cement renders, plasters, suspended ceilings and indoor flooring from all of Spain, as per the requirements indicated by the users. In addition, the causes of these deficiencies are also found, as well as their percentage according to the type of construction they are found in (flats or houses). From the data used, the most typical groups of damages are indicated, as well as a procedure to determine the probability of future damages, according to a weighted matrix classifying the correspondence between 4 descriptors. The knowledge of these results will assist technicians to minimise damages in these types of construction units.

Keywords: Construction anomalies, cement-renders and gypsum-plasters, flooring and tiling, deficiencies.

(*) Fundación-Musaat y Universidad de Extremadura (España).

Persona de contacto/Corresponding author: carreteroayuso@yahoo.es (M.J. Carretero-Ayuso) ORCID: https://orcid.org/o0oo-0002-8082-8996 (M.J. Carretero-Ayuso); https://orcid.org/oooo-0002-1909-9202 (A. Moreno-Cansado)

Cómo citar este artículo/Citation: Carretero-Ayuso, M.J.; Moreno-Cansado, A. (2019). Cuantificación porcentual de los daños existentes en los revestimientos interiores de las viviendas españolas. Informes de la Construcción, 71(555): e303. https://doi. org/10.3989/ic.65059.

Copyright: (C) 2019 CSIC. Este es un artículo de acceso abierto distribuido bajo los términos de la licencia de uso y distribución Creative Commons Reconocimiento 4.0 Internacional (CC BY 4.0). 


\section{INTRODUCCIÓN}

El poder poseer un conocimiento de los valores y porcentajes de presencia de los daños en edificación es una importante forma de retroalimentar el proceso constructivo (1). Éste debe entenderse como un valioso instrumento experimental a utilizar como flujo de datos al realizar un proyecto u obra. De esta manera, podrán minimizarse las deficiencias por parte de los técnicos que intervienen en cada una de las fases de producción.

Hay diversos estudios (2)(3) que han determinado en sus respectivos ámbitos, cuáles son los defectos provenientes de la etapa de diseño, de la etapa de ejecución o de la etapa de uso. Para la investigación realizada por Sá et al. (4), el grupo de causas atribuibles a errores de proyecto es superior a los del proceso de levante y ejecución.

Garcez et al. (5) dan una importancia primordial a la fase de diseño, por la importancia que tiene la misma en la durabilidad a futuro de los elementos constructivos y su repercusión en la vida útil de éstos. A efectos de la planificación del mantenimiento y la estimación de la vida útil, los resultados obtenidos en otros estudios (6) recomiendan que la cuantificación global de daños es interesante hacerla con el uso de herramientas y modelos holísticos, en función de las deficiencias existentes en cada caso.

A diferencia de lo que pueda pensarse, los daños que se dan hoy en día en las construcciones de manera habitual no son grandes colapsos espectaculares, sino otras deficiencias menos críticas como la entrada de agua, la falta de mantenimiento o fisuras no demasiado importantes (7).

Una aspiración que debe tenerse en la construcción de los edificios es la prevención de daños y deficiencias, la cual debe convertirse en una modesta pero legítima contribución a la sostenibilidad y a la seguridad (8). De hecho, el aprender de los fallos se considera desde hace muchos años como una manera de entender los componentes del error humano dentro del proceso constructivo (9). En este sentido, existe un estudio de la Universidad de Sevilla que profundiza en la implementación de un sencillo método de automatización y tipificación de daños materiales en elementos de acabado, como forma de conocimiento y retroalimentación del proceso edificatorio (10).

Hay veces que los problemas de las obras son tan importantes que incluso llegan a la opinión pública a través de los medios de comunicación; aunque esto no suele ser frecuente. Cuando se antepone por encima de todo el diseño, la estética y la visión de la arquitectura cual esculturas urbanas, quedando al mismo tiempo la resolución de las necesidades de uso y construcción en un segundo plano, puede llegarse al desastre. Para Ferguson \& Clayton (11) es imprescindible proporcionar la suficiente información y recomendaciones sobre los principios y procedimientos que son eficaces para mejorar la calidad de los proyectos construidos, de forma que no se reiteren las mismas problemáticas.

Castellano et al. (12) desarrollaron una herramienta para los propietarios de viviendas, que evaluaba la sostenibilidad sobre la base de los impactos sociales, ambientales y económicos, demostrando que aumenta el rendimiento del edificio cuando se implementan criterios sostenibles en los proyectos de vivienda.
Mesa Fernández et al. (13) identificaron y clasificaron los defectos más habituales del control de calidad empleados habitualmente en los edificios, entre otros, los relacionados con los elementos interiores. Haciendo una retrospectiva, conviene reseñar que ya bastante tiempo Mills et al. (14) analizaron las viviendas construidas en Victoria (Australia) entre 1982 y 1997, la naturaleza de los defectos más importantes y el impacto del tipo de contratista y tipo de edificio. Sus resultados siguen siendo extrapolables en muchos aspectos.

Mateus et al. (15) analizaron las ventajas de tabiques ligeros y su contribución al desarrollo de nuevas tecnologías de la pared de separación. La metodología que utilizaron comprendió el análisis del ciclo de vida medioambiental, económica y funcional. Sin embargo, no profundizó en el análisis de deficiencias constructivas relacionadas.

Taggart et al. (16) analizaron las diferencias entre los defectos que se produjeron durante la construcción de 2 a 6 años después de la ocupación inicial. Los defectos encontrados durante la etapa de ocupación son comúnmente conocidos como defectos latentes, la mayoría de las cuales rara vez aparecen en la etapa de construcción,y por lo tanto, rara vez se contabilizan por los proyectistas y los intervinientes en la etapa de construcción. En este trabajo se confirma la necesidad de establecer métodos más eficaces para reducir los defectos latentes.

En lo que respecta al comportamiento de los materiales aislantes utilizados para compartimentar los edificios, una investigación italiana realizó una revisión de los principales materiales comercializados para el sector de la construcción (17), teniendo en cuenta entre otras, las propiedades térmicas, acústicas, la reacción al fuego y la resistencia al vapor de agua.

Gaião et al. (18) clasificaron los defectos en los revestimientos y sus causas más probables. Propusieron un sistema de apoyo a la inspección y diagnóstico que caracterizaba la situación patológica de cada elemento inspeccionado, facilitando la sistematización de las inspecciones incluidas en las actuaciones de mantenimiento periódico y evitando así futuros defectos. Este enfoque, centrándolo en los revestimientos de suelos de madera, ha sido recientemente aplicado por el departamento de ingeniería civil de la Universidad de Lisboa, en una muestra de 35 edificios (19), y también, al estudio de la variabilidad de pruebas in situ destinadas a conocer el envejecimiento natural de revestimientos de paredes enlucidas (20).

Gran parte de las investigaciones consultadas, se han centrado en analizar los defectos observados en un edificio particular o en muestras poco extensas. Otra parte de las investigaciones se basaban en encuestas realizadas a los usuarios de los edificios. Los datos manejados en la investigación que aquí se presentan, se centran en el 100\% de los informes periciales de reclamaciones reales de usuarios, con sentencia firme en España, siendo ésta la principal fortaleza del mismo, pues no se han encontrado precedentes. El objetivo de este artículo es mostrar la recurrencia de los daños existentes en España, dentro del capítulo de revestimientos interiores y en base a las reclamaciones antes indicadas, mostrando los distintos descriptores que intervienen en ello, así como la evaluación general de los resultados obtenidos.

\section{METODOLOGÍA}

El proceso metodológico utilizado en esta investigación toma de base los datos de los partes de siniestros comunicados al 
seguro de responsabilidad civil de los aparejadores y arquitectos técnicos (21). De entre ellos se seleccionaron aquellos que tenían abiertos expedientes judiciales debidos a la reclamación de los usuarios por la existencia de deficiencias constructivas en sus edificios. Estos datos provienen del estudio promovido por la Fundación-Musaat titulado "Análisis estadístico nacional sobre patologías en la edificación” (22), que tomó como periodo de investigación los años 2008-20092010, con el requisito adicional de que se poseyera una resolución judicial en firme sobre los mismos (23).

A partir de aquí, se han realizado las cuantificaciones y valoraciones que en los siguientes apartados se detallan, de tal forma que cada deficiencia fue caracterizada por 4 descriptores, los cuales identifican a cada uno de los 342 casos que fueron determinados.

Descriptor 1 (unidad constructiva). Es el elemento donde se encuentra el daño. En esa investigación corresponden a: 'alicatados y chapados' (AC), 'enfoscados de cemento' (EC), 'falsos techos' (FT), 'guarnecidos de yeso' (GY) y 'solados interiores' (SI). Se trata de 5 unidades constructivas interiores a la envolvente de los edificios (sin incluir las zonas de cimentación, estructura, fachadas y cubiertas).
Descriptor 2 (origen patológico). Se trata de la motivación por la que se puede llegar al daño. Se ha dividido en dos categorías: 'propiedades y características de los materiales' (MTR) y ‘condiciones de disposición y aplicación’ (CDA).

Descriptor 3 (formato constructivo). Es la tipología de edificio en donde han aparecido los daños. Se divide en dos formatos: 'bloques de viviendas' (BLQ) y 'viviendas unifamiliares' (UNF).

Descriptor 4 (modalidad de daño). Es la incidencia o lesión en sí misma. Se caracterizaron las 10 variantes que se indican en la Tabla 1.

\section{RESULTADOS}

\subsection{Unidad constructiva}

Se ha cuantificado el número de casos existentes por cada una de las 5 unidades constructivas estudiadas. Los resultados proporcionan unos valores muy diferentes, de tal forma que se aprecia que existen revestimientos interiores que son muchos más propicios a que se den daños en los mismos (Figura 1). Entre ellos, destacan los 'solados interiores' que obtiene

Tabla 1. Relación, codificación y descripción de la modalidad de daños existentes.

\begin{tabular}{|c|l|l|}
\hline CÓD.-D & \multicolumn{1}{|c|}{ MODALIDAD DE DAÑO } & \multicolumn{1}{c|}{ DESCRIPCIÓN } \\
\hline D-A & Levantamientos y roturas de baldosas & Abombamiento y/o fractura de piezas destinadas al acabado interior \\
\hline D-B & Desconchados y caída de piezas & Astillado o caída de piezas destinadas al acabado interior \\
\hline D-C & Humedad en los materiales de acabados & Afección de agua en las superficies de los materiales de terminación \\
\hline D-D & Manchas o suciedad & Alteración del aspecto visual o superficial de un revestimiento \\
\hline D-E & Fisuras en los materiales de acabados & Rotura superficial del revestimiento de un elemento constructivo \\
\hline D-F & Fisuras modulares & Rotura entre piezas, dimensionalmente iguales \\
\hline D-G & Abertura de juntas entre piezas & Se marcan o abren en exceso las juntas existentes entre piezas \\
\hline D-H & Descuelgues y desprendimientos & Caída o defecto en el método de sustentación de materiales o elementos \\
\hline D-I & Problemas de deslizamiento superficial & Característica superficial de un solado por la que produce riesgo de caídas \\
\hline D-J & La junta con paramentos queda marcada & Defecto visible del encuentro entre el falso techo y la pared adyacente \\
\hline
\end{tabular}
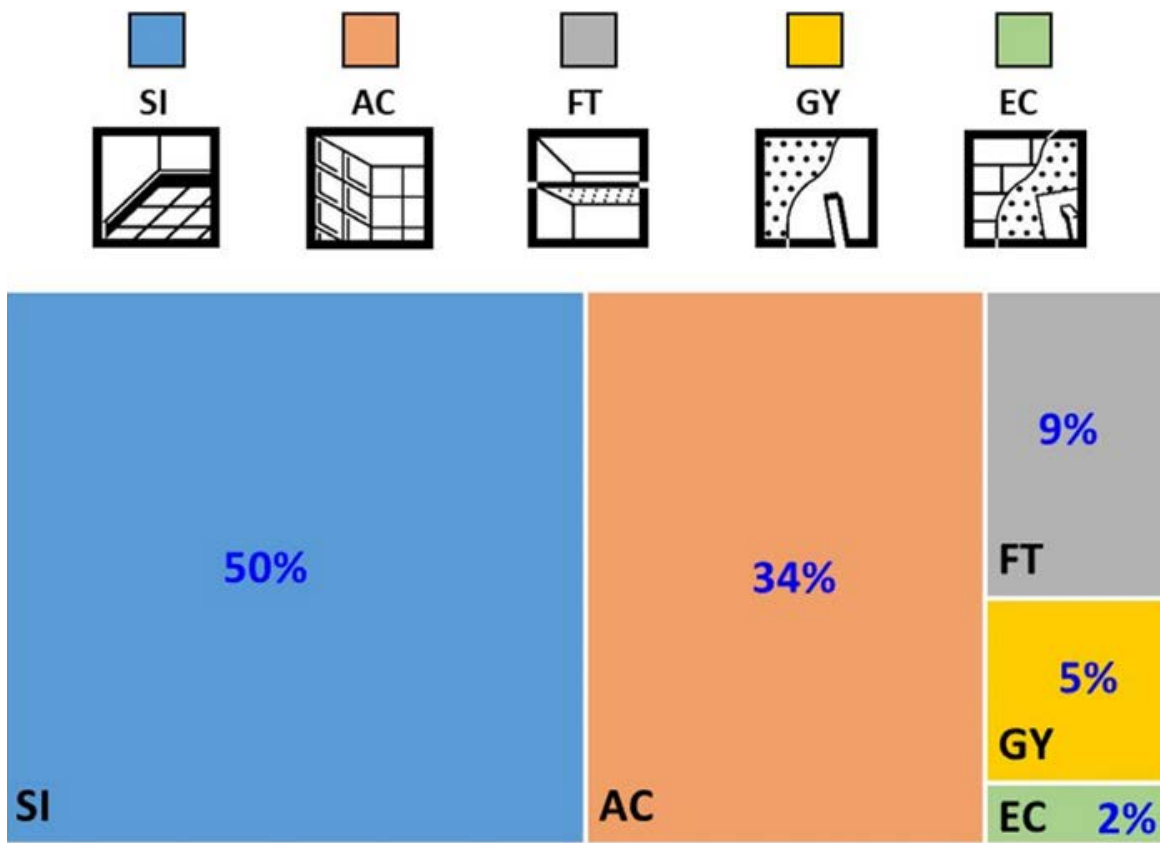

Figura 1. Porcentaje de casos por cada unidad constructiva 
la mitad exacta de toda la muestra ( $\mathrm{SI}=171$ casos). Le sigue 'alicatados y chapados' ( $\mathrm{AC}=117$ casos) con el $34 \%$. A mucha distancia, se encuentran los 'falsos techos' ( $\mathrm{FT}=30$ casos), los 'guarnecidos de yeso' (GY=18 casos) y finalmente los 'enfoscados de cemento' ( $\mathrm{EC}=6$ casos).

\subsection{Origen patológico}

Se ha determinado el motivo causal por el que aparecían los daños (origen patológico). Se apreció que en la mayoría de los casos (excepto uno) el origen era por causas relacionadas con el material (MTR) en un porcentaje que era cercano o mayor a las 2/3 partes. Es también interesante destacar que dos unidades constructivas tenían la totalidad de su origen patológico en una de las dos categorías existentes: FT con $\mathrm{CDA}=100 \%$ $\mathrm{y}$ EC con $\mathrm{MTR}=100 \%$.

\subsection{Formato constructivo}

Efectuando una distribución de los casos en función del formato constructivo en donde aparecieron las deficiencias (Figura 2), se aprecia una clara preponderancia de los que se dan en los 'bloques de viviendas' ( $\mathrm{BLQ}=208$ casos) pues concentran el $61 \%$ de las situaciones, mientras que las 'viviendas unifamiliares' (UNF=134 casos) obtuvieron el 39\%. Si el análisis se hace por cada una de las unidades constructivas, los 'bloques de viviendas' tienen una presencia porcentual análoga en todas ellas, exceptuando en SI donde es algo menor.

Para conocer el desglose de la modalidad de daño y la categoría de origen patológico, por cada uno de los dos formatos constructivos, se ha confeccionado la Tabla 2, en donde se proporciona el porcentaje de casos respecto al valor suma de cada modalidad de daño, así como con respecto al cómputo general de la población estudiada.
De la lectura de esta tabla, se aprecia que el mayor porcentaje para una modalidad daño se obtiene con un $78 \%$, referido a $\mathrm{D}-\mathrm{H} / \mathrm{CDA} / \mathrm{BLQ}$ (descuelgues y desprendimientos debidos a condiciones de disposición y aplicación, y situados en bloques de viviendas). Le sigue con un $67 \%$, referido a D-I/ MTR/BLQ (problemas de deslizamiento supericial debidos a propiedades y características de los materiales, y situados en bloques de viviendas). Por el contrario, el menor porcentaje de daños (con un o\%) se obtiene en D-C/MTR-BLQ, D-C/MTR-UNF， D-F/MTR-BLQ， D-F/MTR-UNF， D-H/ MTR-BLQ, D-H/MTR-UNF, D-J/MTR-BLQ, D-J/MTR-UNF y D-I/MTR-BLQ, D-I/MTR-UNF. De las 10 situaciones antes indicadas, se constata que hay 4 modalidades de daños que solo tienen presencian en una de las dos categorías de origen patológico, en este caso, en CDA (D-C, D-F, D-H y D-J). Por el contario, hay otra (D-I) que tiene concentrados todos sus casos en MTR.

En todas las situaciones presentes en la tabla, los valores de BLQ son mayores a los de UNF, excepto en D-D/CDA y D-J/ CDA que están igualados, y en D-A/MTR que el mayor porcentaje se da en UNF. Con todos estos resultados, los técnicos pueden hacer un análisis a futuro para conocer cuál de las situaciones concretas de sus proyectos y de sus obras pueden conducir a estas distribuciones, de forma que una vez analizadas exista una retroalimentación que permita la reducción de daños en sus siguientes actuaciones.

\subsection{Modalidad de daño}

La modalidad de daño ha sido el descriptor más numeroso y con más variedad. De entre las 10 variedades existentes, hay dos con una clara preponderancia entre las demás. La primera ha sido 'levantamientos y roturas de baldosas' (D-A) con un $32 \%$ de los casos, y 'desconchados y caída de piezas' (D-B) con un 22\%. Le sigue 'humedad en los materiales de

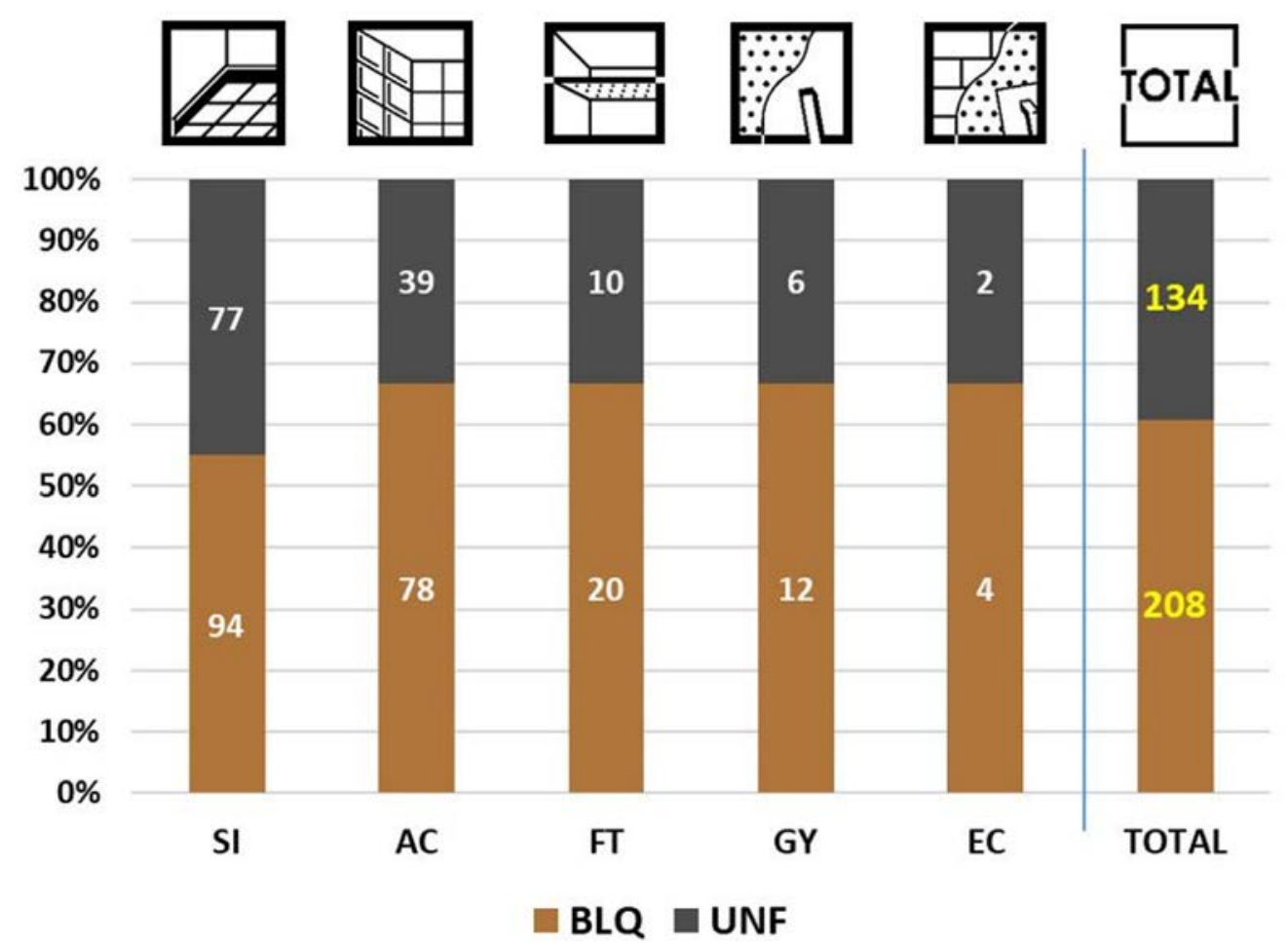

Figura 2. Distribución del número de casos según el formato constructivo. 
acabados' (D-C) y 'manchas o suciedad' (D-D) con un $14 \%$ y un $13 \%$, respectivamente.

Se han encontrado 4 daños (D-G, D-H, D-I y D-J) que tienen un valor individual menor al $5 \%$ y que en conjunto suman el 9\% de los casos, según se muestra en la Figura 3. Entre ellos, el más numeroso es 'abertura de juntas entre piezas' (D-G).
Para tener una idea más clara y resumida de todas las modalidades de daño, éstos se han agrupado según su similitud (analogía tipológica de daños), según se muestra en la Tabla 3. De esta forma, se ha pasado de 10 modalidades de daños a 4 'grupos de daños agrupados', los cuales se han denominado 'ABERTURAS', 'HUMEDADES', 'SUPERFICIES' y 'ROTURAS'. El primero de ellos concentra la mayor parte de los daños (209 $\rightarrow 61,11 \%)$, de los cuales 154 casos correspon-

Tabla 2. Porcentaje de daños según el origen patológico y el formato constructivo.

\begin{tabular}{|c|c|c|c|c|}
\hline \multirow{2}{*}{$\begin{array}{l}\text { MODALIDAD DE } \\
\text { DAÑO }\end{array}$} & \multirow{2}{*}{$\begin{array}{c}\text { ORIGEN } \\
\text { PATOLÓGICO }\end{array}$} & \multicolumn{3}{|c|}{ FORMATO CONSTRUCTIVO } \\
\hline & & BLQ & UNF & TOTAL \\
\hline \multirow{4}{*}{ D-A } & \multirow{2}{*}{ MTR } & 33 & 34 & 67 \\
\hline & & $(10,5)$ & $(10,8)$ & $(21,3)$ \\
\hline & \multirow{2}{*}{ CDA } & 22 & 11 & 33 \\
\hline & & $(7,0)$ & $(3,8)$ & $(10,8)$ \\
\hline \multirow{4}{*}{ D-B } & \multirow{2}{*}{ MTR } & 65 & 30 & 95 \\
\hline & & $(14,0)$ & $(6,4)$ & $(20,5)$ \\
\hline & \multirow{2}{*}{$\mathrm{CDA}$} & 4 & 1 & 5 \\
\hline & & $(0,9)$ & $(0,3)$ & $(1,2)$ \\
\hline \multirow{4}{*}{$\mathrm{D}-\mathrm{C}$} & \multirow{2}{*}{ MTR } & o & o & o \\
\hline & & $(0,0)$ & $(0,0)$ & $(0,0)$ \\
\hline & \multirow{2}{*}{$\mathrm{CDA}$} & 61 & 39 & 100 \\
\hline & & $(8,8)$ & $(5,6)$ & $(14,3)$ \\
\hline \multirow{4}{*}{ D-D } & \multirow{2}{*}{ MTR } & 50 & 40 & 90 \\
\hline & & $(6,4)$ & $(5,3)$ & $(11,7)$ \\
\hline & \multirow{2}{*}{ CDA } & 5 & 5 & 10 \\
\hline & & $(0,6)$ & $(0,6)$ & $(1,2)$ \\
\hline \multirow{4}{*}{ D-E } & \multirow{2}{*}{ MTR } & 63 & 21 & 84 \\
\hline & & $(3,5)$ & $(1,2)$ & $(4,7)$ \\
\hline & \multirow{2}{*}{$\mathrm{CDA}$} & 11 & 5 & 16 \\
\hline & & $(0,6)$ & $(0,3)$ & $(0,9)$ \\
\hline \multirow{4}{*}{ D-F } & \multirow{2}{*}{ MTR } & 0 & o & 0 \\
\hline & & $(0,0)$ & $(0,0)$ & $(0,0)$ \\
\hline & \multirow{2}{*}{$\mathrm{CDA}$} & 61 & 39 & 100 \\
\hline & & $(3,2)$ & $(2,0)$ & $(5,3)$ \\
\hline \multirow{4}{*}{ D-G } & \multirow{2}{*}{ MTR } & 36 & 29 & 65 \\
\hline & & $(1,5)$ & $(1,2)$ & $(2,6)$ \\
\hline & \multirow{2}{*}{$\mathrm{CDA}$} & 21 & 14 & 35 \\
\hline & & $(0,8)$ & $(0,6)$ & $(1,4)$ \\
\hline \multirow{4}{*}{ D-H } & \multirow{2}{*}{ MTR } & 0 & o & 0 \\
\hline & & $(0,0)$ & $(0,0)$ & $(0,0)$ \\
\hline & CDA & 78 & 22 & 100 \\
\hline & CDA & $(2,0)$ & $(0,6)$ & $(2,6)$ \\
\hline & MTR & 67 & 33 & 100 \\
\hline$D_{-1}$ & & $(0,6)$ & $(0,3)$ & $(0,9)$ \\
\hline$D-1$ & $C D A$ & $\mathrm{O}$ & o & $\mathrm{O}$ \\
\hline & CDA & $(0,0)$ & $(0,0)$ & $(0,0)$ \\
\hline & MTR & 0 & 0 & 0 \\
\hline D-I & IVIK & $(0,0)$ & $(0,0)$ & $(0,0)$ \\
\hline & CDA & 50 & 50 & 100 \\
\hline & CDA & $(0,3)$ & $(0,3)$ & $(0,6)$ \\
\hline
\end{tabular}




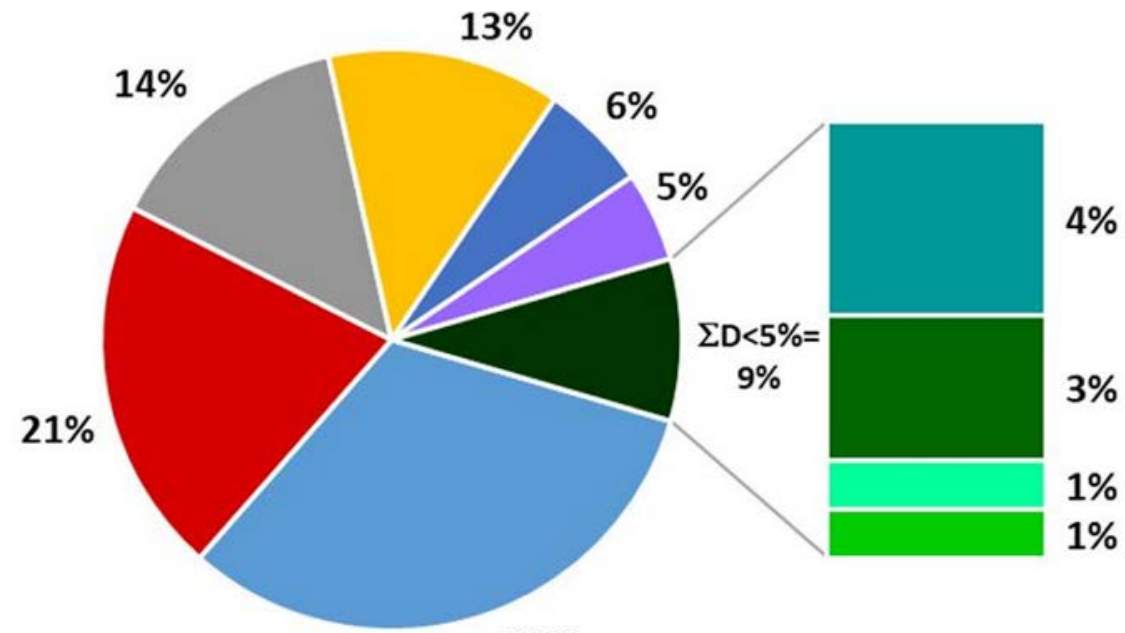

$32 \%$

\begin{tabular}{|c|c|c|c|c|c|c|c|c|c|}
\hline$=\mathrm{D}-\mathrm{A}$ & $\square \mathrm{D}-\mathrm{B}$ & $=\mathrm{D}-\mathrm{C}$ & $=\mathrm{D}-\mathrm{D}$ & $=\mathrm{D}-\mathrm{E}$ & D-F & D-G & D-H & $=\mathrm{D}-\mathrm{I}$ & $=$ D-J \\
\hline 110 & 74 & 40 & 44 & rat & 10 & 14 & 5 & 3 & \\
\hline
\end{tabular}

Figura 3. Porcentaje y número de casos según la modalidad de daños.

Tabla 3. Agrupación de daños por similitud.

\begin{tabular}{|c|c|c|c|c|c|c|c|}
\hline \multirow{3}{*}{ CONCEPTO } & \multirow{3}{*}{ GRUPO } & \multirow{3}{*}{ DAÑOS AGRUPADOS } & \multicolumn{5}{|c|}{ NÚMERO DE CASOS } \\
\hline & & & \multicolumn{2}{|c|}{$\begin{array}{c}\text { Ordenados por } \\
\text { Descriptor } 2\end{array}$} & \multicolumn{2}{|c|}{$\begin{array}{l}\text { Ordenados por } \\
\text { Descriptor } 3\end{array}$} & \multirow{2}{*}{ TOTAL } \\
\hline & & & CDA & MTR & BLQ & UNF & \\
\hline $\begin{array}{l}\text { Aberturas, } \\
\text { desplazamientos y } \\
\text { desprendimientos }\end{array}$ & ABERTURAS & $\mathrm{D}-\mathrm{A}+\mathrm{D}-\mathrm{B}+\mathrm{D}-\mathrm{G}+\mathrm{D}-\mathrm{H}+\mathrm{D}-\mathrm{J}$ & 55 & 154 & 127 & 82 & 209 \\
\hline $\begin{array}{l}\text { Existencia de fugas o de } \\
\text { humedad }\end{array}$ & HUMEDADES & $\mathrm{D}-\mathrm{C}$ & 46 & 3 & 30 & 19 & 49 \\
\hline $\begin{array}{l}\text { Características de } \\
\text { superficie del Material }\end{array}$ & SUPERFICIES & $\mathrm{D}-\mathrm{D}+\mathrm{D}-\mathrm{I}$ & 4 & 43 & 26 & 21 & 47 \\
\hline Roturas y fracturas & ROTURAS & $\mathrm{D}-\mathrm{E}+\mathrm{D}-\mathrm{F}$ & 21 & 16 & 25 & 12 & 37 \\
\hline
\end{tabular}

den a MTR y 55 casos a CDA (si se ordenan según el descriptor 2), mientras que 127 casos corresponden a bloques de viviendas -BLQ- y 82 casos a viviendas unifamiliares -UNF- (si se ordenan según el descriptor 3 ).

Como se aprecia, en dicha tabla los 4 grupos de daños agrupados dependen íntimamente de la modalidad de daño en sí, y éstos a su vez dependen del formato constructivo donde se producen. Realizando la prueba de chi-cuadrado de Pearson, obtendríamos por tanto que se trata de variables/parámetros no independientes (existe una interrelación numérica que corresponde a la correlación constructiva que efectivamente existe entre todos ellos).

Para profundizar en la casuística, estos 'daños agrupados' se han combinado numérica y simultáneamente con el 'origen patológico' (descriptor 2) y con el 'formato constructivo' (descriptor 3), al objeto de verificar cual es la combinación más numerosa (Figura 4). Bajo los tres parámetros antes descritos, se han encontrado 4 'relaciones tri-laterales' más recurrentes: ABERTURAS-BLQ-MTR (9o casos), ABERTURASUNF-MTR (64 casos), HUMEDADES-BLQ-CDA ( 28 casos) y SUPERFICIES-BLQ-MTR (24 casos).

\subsection{Determinación de las categorías de probabilidad ante requerimientos}

En la Tabla 4 se han determinado las categorías de probabilidad ante el requerimiento por parte de los usuarios, en función de la naturaleza y frecuencia de las interrelaciones 'daño y origen patológico', según la siguiente sistemática:

Etapa 1- Graduación en función del grado de problemática que representa la modalidad de daño (D-x) y su origen patológico (CDA o MTR). Para los daños, la puntuación se basa en el grado de molestia o percepción de insatisfacción de los usuarios o propietarios debido a la presencia de las mismas (puntos: 1,3 o 5 ). Para el origen patológico, la puntuación se hace según el grado de importancia técnica o constructiva que representa dicho origen (puntos: 2 o 4 ). Para la categorización y puntuación de estos aspectos se ha recurrido a un grupo de 7 expertos, los cuales han llevado a cabo dicha clasificación, según queda expresado en el tramo superior de la mencionada tabla.

Etapa 2- Nivel de severidad conjunta, la cual se define como la combinación numérica de los dos grados definidos en la 


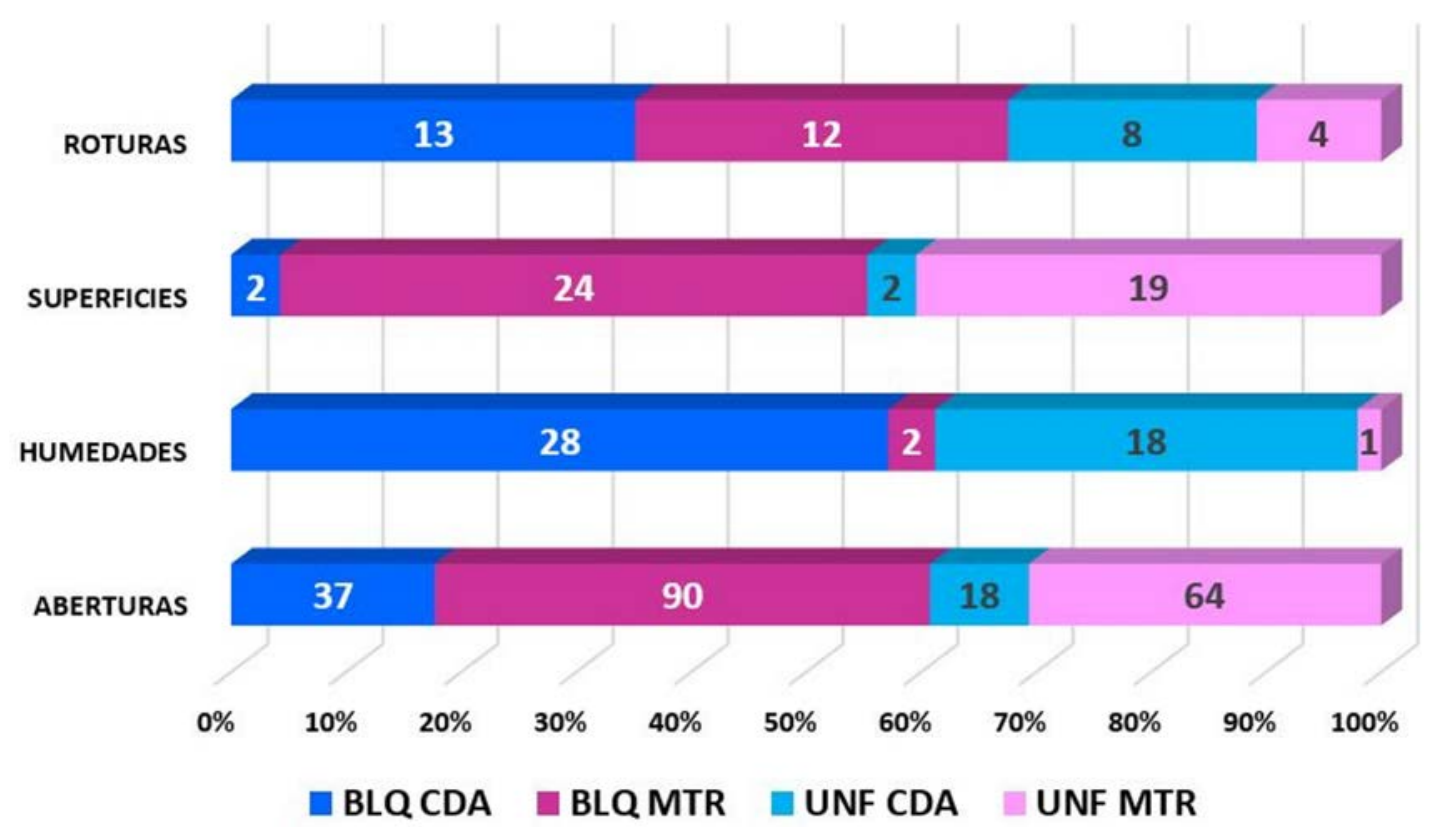

Figura 4. Distribución de casos según la agrupación de daños, el formato constructivo y el origen patológico.

Tabla 4. Determinación de la probabilidad ante requerimientos en función de los valores obtenidos en la investigación.

\begin{tabular}{|c|c|c|c|c|c|c|c|c|c|c|c|c|}
\hline ETAPAS & \multicolumn{2}{|c|}{ CONCEPTO } & \multicolumn{10}{|c|}{ VALORES } \\
\hline \multirow{5}{*}{$\begin{array}{l}\text { Graduación en } \\
\text { función el } \\
\text { grado de } \\
\text { problemática } \\
\text { (ETAPA 1) }\end{array}$} & \multicolumn{2}{|c|}{ Tipo de puntuación } & \multicolumn{10}{|c|}{ Valor según cada parámetro } \\
\hline & \multirow{2}{*}{\multicolumn{2}{|c|}{$\begin{array}{l}\text { Puntuación según } \\
\text { molestia a los usuarios }\end{array}$}} & $\mathrm{D}-\mathrm{A}$ & D-B & $\mathrm{D}-\mathrm{C}$ & $\mathrm{D}-\mathrm{D}$ & $\mathrm{D}-\mathrm{E}$ & D-F & $\mathrm{D}-\mathrm{G}$ & $\mathrm{D}-\mathrm{H}$ & D-I & D-J \\
\hline & & & 5 & 5 & 3 & 1 & 3 & 3 & 1 & 5 & 5 & 1 \\
\hline & \multirow{2}{*}{\multicolumn{2}{|c|}{$\begin{array}{l}\text { Puntuación según } \\
\text { importancia técnica }\end{array}$}} & MTR & $\mathrm{CDA}$ & & & & & & & & \\
\hline & & & 2 & 4 & & & & & & & & \\
\hline \multirow{4}{*}{$\begin{array}{l}\text { Nivel de } \\
\text { severidad } \\
\text { conjunta } \\
\text { entre el daño } \\
\text { y el origen } \\
\text { patológico } \\
\text { (ETAPA 2) }\end{array}$} & \multirow{2}{*}{\multicolumn{2}{|c|}{$\begin{array}{l}\text { Puntuación combinada } \\
\text { por cada interrelación }\end{array}$}} & \multicolumn{10}{|c|}{ Modalidad de daño } \\
\hline & & & D-A & D-B & $\mathrm{D}-\mathrm{C}$ & D-D & D-E & $\mathrm{D}-\mathrm{F}$ & D-G & $\mathrm{D}-\mathrm{H}$ & D-I & D-J \\
\hline & \multirow{2}{*}{$\begin{array}{l}\text { Origen } \\
\text { patológico }\end{array}$} & MTR & 10 & 10 & 6 & 2 & 6 & 6 & 2 & 10 & 10 & 2 \\
\hline & & $\mathrm{CDA}$ & 20 & 20 & 12 & 4 & 12 & 12 & 4 & 20 & 20 & 4 \\
\hline \multirow{4}{*}{$\begin{array}{l}\text { Matriz de } \\
\text { correlación } \\
\text { e intensidad } \\
\text { entre } \\
\text { el daño y } \\
\text { el origen } \\
\text { patológico } \\
\text { (ETAPA 3) }\end{array}$} & \multirow{2}{*}{\multicolumn{2}{|c|}{$\begin{array}{l}\text { Frecuencia de cada } \\
\text { interrelación }\end{array}$}} & \multicolumn{10}{|c|}{ Modalidad de daño } \\
\hline & & & $\mathrm{D}-\mathrm{A}$ & D-B & $\mathrm{D}-\mathrm{C}$ & D-D & D-E & $\mathrm{D}-\mathrm{F}$ & D-G & $\mathrm{D}-\mathrm{H}$ & D-I & D-J \\
\hline & \multirow{2}{*}{$\begin{array}{l}\text { Origen } \\
\text { patológico }\end{array}$} & MTR & 0,67 & 0,95 & o & 0,9 & 0,84 & o & 0,65 & o & 1 & o \\
\hline & & $\mathrm{CDA}$ & 0,33 & o,05 & 1 & 0,1 & 0,16 & 1 & 0,35 & 1 & o & 1 \\
\hline \multirow{4}{*}{$\begin{array}{l}\text { Matriz de } \\
\text { probabilidad } \\
\text { ponderada ante } \\
\text { requerimientos } \\
\text { (ETAPA 4) }\end{array}$} & \multirow{2}{*}{\multicolumn{2}{|c|}{$\begin{array}{c}\text { Matriz de correlación } \\
\text { en función de la } \\
\text { puntuación }\end{array}$}} & \multicolumn{10}{|c|}{ Modalidad de daño } \\
\hline & & & $\mathrm{D}-\mathrm{A}$ & D-B & $\mathrm{D}-\mathrm{C}$ & $\mathrm{D}-\mathrm{D}$ & $\mathrm{D}-\mathrm{E}$ & $\mathrm{D}-\mathrm{F}$ & $\mathrm{D}-\mathrm{G}$ & $\mathrm{D}-\mathrm{H}$ & D-I & D-J \\
\hline & \multirow{2}{*}{$\begin{array}{l}\text { Origen } \\
\text { patológico }\end{array}$} & MTR & 6,70 & 9,50 & 0,00 & 1,80 & 5,04 & 0,00 & 1,30 & 0,00 & 10,00 & 0,00 \\
\hline & & $\mathrm{CDA}$ & 6,60 & 1,00 & 12,00 & 0,40 & 1,92 & 12,00 & 1,40 & 20,00 & 0,00 & 4,00 \\
\hline \multirow{6}{*}{$\begin{array}{l}\text { Categorías de } \\
\text { probabilidad } \\
\text { ante } \\
\text { requerimientos } \\
\text { por parte de } \\
\text { los usuarios } \\
\text { (ETAPA 5) }\end{array}$} & Categoría & Código & \multicolumn{10}{|c|}{ Determinación según los valores de la probabilidad de requerimientos } \\
\hline & Roja & $\mathrm{R}$ & \multicolumn{3}{|c|}{$\mathrm{R}: \mathrm{P}>15$} & \multicolumn{4}{|c|}{ (1 interrelación) } & \multirow{5}{*}{\multicolumn{3}{|c|}{$\begin{array}{c}\mathrm{P}=\text { Probabilidad de } \\
\text { requerimientos } \\
\text { (valor según matriz } \\
\text { de probabilidad } \\
\text { ponderada en Etapa 4) }\end{array}$}} \\
\hline & Naranja & $\mathrm{N}$ & \multicolumn{3}{|c|}{$\mathrm{N}: 10<\mathrm{P} \leq 15$} & \multicolumn{4}{|c|}{ (2 interrelaciones) } & & & \\
\hline & Amarilla & A & & $: 5<\mathrm{P} \leq$ & & \multicolumn{4}{|c|}{ (4 interrelaciones) } & & & \\
\hline & Verde & $\mathrm{V}$ & & $: \mathrm{O}<\mathrm{P}$ & & \multicolumn{4}{|c|}{ (8 interrelaciones) } & & & \\
\hline & Blanca & B & \multicolumn{3}{|c|}{$\mathrm{B}: \mathrm{P}=\mathrm{O}$} & \multicolumn{4}{|c|}{ (5 interrelaciones) } & & & \\
\hline
\end{tabular}


Etapa 1. En el segundo tramo de la tabla se incluyen los resultados, los cuales se obtienen multiplicando cada categoría de origen patológico por cada modalidad de daño.

Etapa 3- Se ha cuantificado cada una de las 10 combinaciones posibles de interrelación entre las 10 modalidades de daño y las 2 categorías de origen patológico (valores expresados respecto al valor total de cada modalidad de daño). En función de estos porcentajes de presencia, se ha establecido una matriz de correlación e intensidad entre los daños y sus orígenes (los resultados se muestran en el tercer tramo de la Tabla 4).

Etapa 4- Se crea una matriz de riesgo ponderado sobre los requerimientos, la cual resulta de multiplicar el nivel de severidad conjunta por la matriz de correlación e intensidad. A cada valor de esta matriz se le denominará 'probabilidad de requerimientos' $(\mathrm{P})$. Los resultados quedan expresados en el cuarto tramo de la Tabla 4 y muestran lo extendido e intenso que puedan estar los casos determinados en la investigación, en función del origen patológico de la que provengan y del daño en que intervengan. De esta manera, el valor de $\mathrm{P}$ cuantifica numéricamente la posibilidades de que un usuario inicie un requerimiento judicial por la presencia de una daño, según la problemática concreta de la que se trate.

Etapa 5- Se establecen 5 categorías de probabilidad ante requerimientos por parte de los usuarios, en función de los valores obtenidos por P en la etapa anterior. Según la horquilla de valores obtenidas, se establacen las categorías Roja (R), Naranja (N), Amarilla (A), Verde (V) y Blanca (B). En base a ellas, se visualiza en el último tramo de la tabla, cuáles factores de interrelación ‘daño-origen patológico' son más recurrentes. Estas categorías cuantifican en 5 escalas todo el proceso de las etapas anteriores.

Como resultado de estos cálculos en cinco etapas, puede observarse que en la mayoría de las ocasiones las interrelaciones 'daño-orígenes patológicos' tienen una baja presencia (categoría $\mathrm{B}$ y V) y son las que más se repiten (con 8 y 5 interrelaciones, respectivamente). Por el contrario, la categoría que agrupa el mayor valor, y por tanto la mayor probabilidad ante requerimientos ( $\mathrm{R}: \mathrm{P}>15)$, se da en solo una ocasión: 'descuelgues y desprendimientos motivados por las condiciones de disposición y aplicación' $(D-H / C D A=2 O, O O)$. En segundo lugar, se encuentran -con dos interrelaciones e igualdas con P:12,00-: 'humedad en los materiales de acabados motivadas por las condiciones de disposición y aplicación' (D-C/ $C D A)$ y 'fisuras modulares motivadas por las condiciones de disposición y aplicación' ( $D-F / C D A)$. Por el contario, los menores valores se obtienen (P:O) en D-C/MTR, D-F/MTR, D-H/MTR, D-I/CDA y D-J/MTR.

\section{DISCUSIÓN}

\subsection{La calidad de la mano de obra en España}

El sector de la construcción en España supuso el 10,1\% del PIB español en la época del boom inmobiliario. Ahora, en estos últimos años apena supone el 5,1\% (24). Antes, a los operarios no les interesaba formarse porque con lo que ganaban y el trabajo que había nadie se lo exigía. Actualmente, se está construyendo gran parte de las promociones sin que exista suficiente mano de obra especializada, después de que las 2/3 partes del empleo anteriormente existente haya sido destruido por la crisis (25). Esta situación puede suponer el volver a caer en un riesgo importante de reiteración de deficiencias de obra, por falta de cualificación de los operarios, entre otros factores. En España es preciso tener un plan estratégico que haga imprescindible el tener un conocimiento y una cualificación reconocida para los distintos oficios, si no se quieren gastar grandes cantidades en reparar las deficiencias debidas a la no-calidad.

\subsection{Lectura de los resultados obtenidos}

En función de los valores obtenidos en esta investigación se pueden extraer una serie de lecciones aprendidas que ayudarían a los técnicos a conocer cuáles son las situaciones y elementos más conflictivos dentro de los revestimientos interiores.

Como unidad constructiva, deberá ponerse especial cuidado en supervisar los procesos de puesta en obra de los 'solados interiores' puesto que en ellos existe una mayor posibilidad estadística de que a futuro existan deficiencias. En ellos suele ser problemático la adherencia entre las baldosas y el soporte base (especialmente cuando son cerámicas). La falta o poco espesor de las juntas entre piezas suele ser también una situación habitual en España.

Es necesario que se incrementen los controles que tienen que ver con las características de los materiales a utilizar en los propios revestimientos, o también, acotar las consecuencias de las retracciones hidráulicas de las unidades constructivas en las que el cemento esté presente. Respecto a las condiciones de disposición y aplicación, hay algunos aspectos que suelen ser repetitivos en la aparición de las deficiencias, como son: insuficiente humectación del elemento soporte, espesor escaso del material que se aplica, sellados inexistentes, defectos de acabado, etc... El deseo de algunos proyectistas a no marcar las juntas o a que éstas sean del menor espesor posible, es también un factor favorecedor de problemáticas; consideración análoga al hecho de que en muchos proyectos no se prescriban las formas de aplicación que posibiliten una adecuada adherencia entre los materiales y sus soportes, a que se incluyan sistemáticas de forma incompleta o a que se omitan muchos detalles constructivos.

La supervisión y reducción de estas situaciones llevará a que se minimicen los casos de daños en los edificios. En la Figura 5 se incluyen cuatro casos de deficiencias tomadas durante la ejecución de unas obras.

\subsection{Otras consideraciones}

Karim et al. (26) comprobaron que el exceso de subcontratación aumenta los errores en la construcción de un edificio; consideraron que una forma de reducir la incidencia de defectos en el proceso constructivo debía centrarse sobre el control de dicha subcontratación. Este aspecto puede ser extrapolable al caso de España, en la que es habitual subcontratar gran parte de las tareas.

Otra manera de disminuir el número de incidencias sería implementar procedimientos de gestión interna de calidad como la norma ISO-9001:2008 o el modelo de calidad EFQM, lo que además genera beneficios en su sistema organizativo y rendimiento económico. 


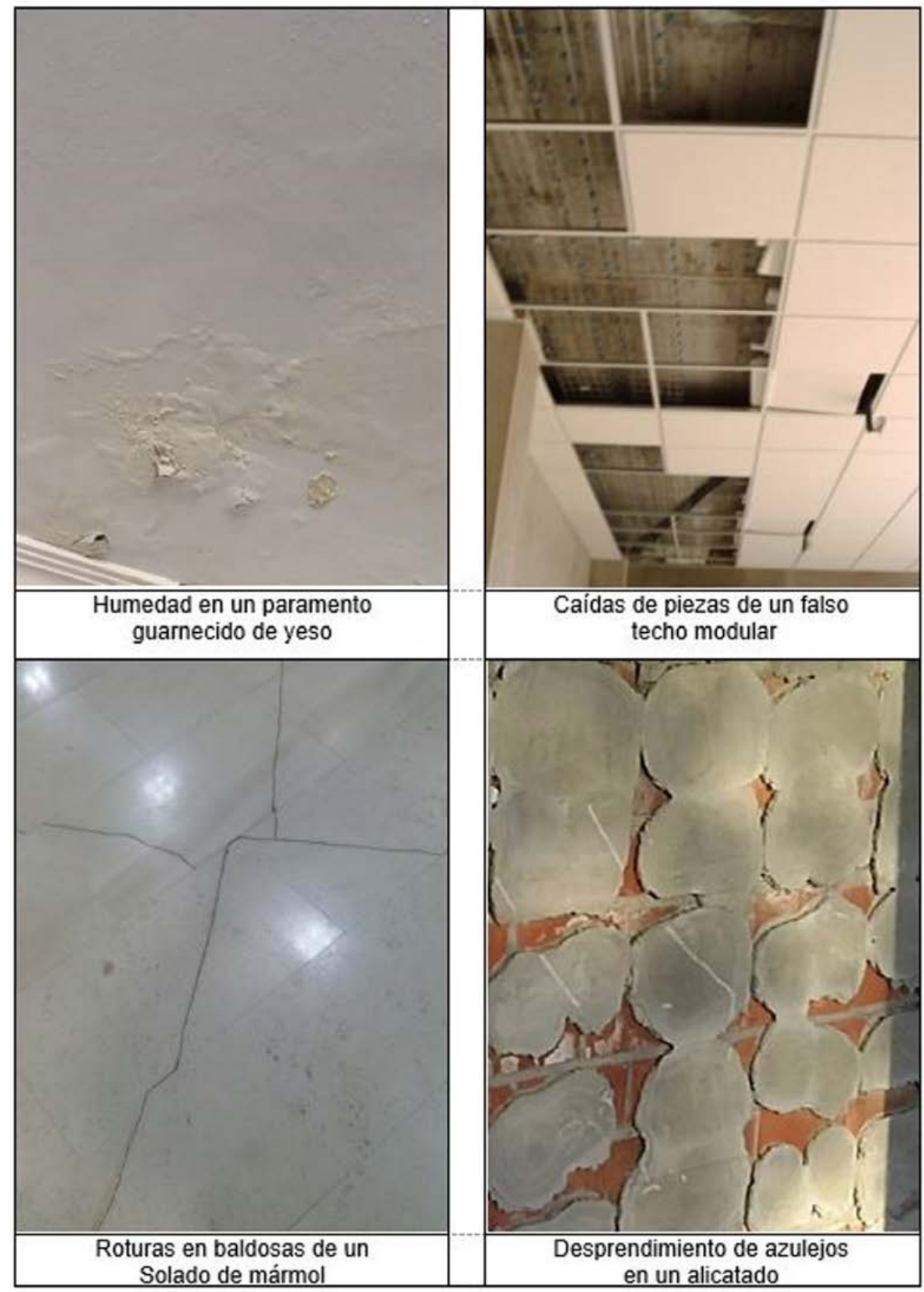

Figura 5. Casos de ejemplo con distintas deficiencias en cuatro unidades constructivas. Fotos realizadas por Manuel J. Carretero-Ayuso.

Hay que recordar que ya en siglos pretéritos había quien en sus tratados de construcción le dedicaba espacio a este tema de los defectos constructivos. De hecho, un importante arquitecto de la época del neoclasicismo, en uno de sus libros dedicó un capítulo a ello, en donde puede leerse: "se pueden reducir a cuatro los tipos de faltas, errores ofraudes en la construcción de una obra: por malicia y de mala fe, por ignorancia, por descuido y aquellos cometidos sin malicia ni ignorancia” (27). Por su parte, Andrea Paladio dijo en su primer Libro de Arquitectura (capítulo 1): "se debe también atender a la calidad y bondad de los materiales, a fin de elegir los mejores; en esto, nos ayudará mucho la experiencia tomada de las obras construidas por otros, pues advertidos por ellas podremos determinar fácilmente lo que a nuestra necesidad más acomoda” (28).
Adoptando una clasificación simplificada y adaptada en la escala de Parker, para el 'camino de la gestión de seguridad y salud', podríamos establecer tres niveles en la implicación por la calidad de la construcción y en la reducción de fallos y errores en todo este sistema productivo. El esquema que utilizan normalmente muchas empresas constructoras y de oficinas de proyecto, respecto a la existencia y resolución de deficiencias en la construcción suele ser de tipo 'reactivo' (solo se actúa cuando se ha tenido previamente un problema en un proyecto previo, de tal forma que se intenta no caer en los mismos errores). Los que están un poco más concienciados y les preocupa la legalidad utilizan un modo 'formalista' (incluyen en sus procedimientos la necesidad de cumplir cierta legislación o estándares en el diseño o ejecución de sus activi- 
dades para intentar quedar exonerados de culpa). Finalmente, los realmente comprometidos con aumentar la calidad, minimizar fallos y rentabilizar el proceso, adoptan una visión general de tipo ‘proactivo' en la cual toda la organización y las personas que lo componen participan en el proceso de mejora continua, preocupándose y esforzándose por la prevención, la seguridad y la eficacia de los procesos.

\section{CONCLUSIONES}

Las conclusiones principales obtenidas en este trabajo, a partir de los resultados obtenidos son las siguientes:

- La unidad constructiva donde se da el mayor número de daños son los 'solados interiores' (SI=50\%), seguido de los 'alicatados y chapados' ( $\mathrm{AC}=34 \%)$.

- Estudiado el origen patológico de cada uno de los 342 casos analizados, en la mayor parte de las ocasiones tienen que ver con las “propiedades y características de los materiales' $(\mathrm{MTR}=211$ vs. $\mathrm{CDA}=131)$.

- En función del formato constructivo, los daños tienen una mayor presencia numérica en los 'bloques de viviendas' (BLQ=208) que en las 'viviendas unifamiliares' (UNF=134).

- La modalidad de daño más recurrente es 'levantamientos y roturas de baldosas' ( $\mathrm{D}-\mathrm{A}=32 \%)$ seguida de 'desconchados y caída de piezas' ( $\mathrm{D}-\mathrm{B}=\mathbf{2 2 \%}$ ).

- Según los tipos de ‘daños agrupados’ (daños similares entre sí), el conjunto con más recurrencia es ABERTURAS (209 casos).

- Se han determinado 5 categorías de probabilidad ante requerimientos judiciales, determinados en función de la molestia a los usuarios y la importancia técnica. La interrelación con mayor probabilidad es 'descuelgues y desprendimientos motivados por las condiciones de disposición y aplicación' (D-H/CDA=20,00).
Como resumen global, podemos hacer la siguiente reflexión en relación al análisis efectuado y las casuísticas de otros estudios consultados: El sector de la construcción español es uno de los sectores económicos donde más problemas de producción y detección de incidencias post-entrega hay (29). Esta realidad señala una evidente insatisfacción del destinatario final, pero también muestra que existen ciertos aspectos constructivos que tendrían que ser mejorados. A este hecho, se añade que los usuarios no siempre perciben por igual la calidad de los materiales, manejan conceptos distintos a los técnicos facultativos y sus valoraciones pueden ser influenciables por aspectos estéticos o funcionales (30). El estudio cualitativo-cuantitativo de las reclamaciones que aquí se presentan, es una forma adecuada para entender la situación en España y conocer así qué daños suelen ser los más habituales en los revestimientos interiores.

Son prácticamente inexistentes los estudios científicos de construcción realizados a partir de dictámenes judiciales, y menos los que se hacen a partir de las sentencias firmes de los tribunales de justicia, dada la dificultad en acceder a esas sentencias, y además, a conseguir el 100\% de todos los casos existentes en un país, a través de los informes periciales en los que se basaron esas sentencias. A pesar de esa dificultad, este trabajo se basa en dichos parámetros judiciales y en ese tipo de datos, lo que permite una nueva aportación al conocimiento de la ciencia mediante esta metodología.

\section{AGRADECIMIENTOS}

El presente trabajo se ha desarrollado dentro del Plan de Actuación que se aprobó en 2011 en la Fundación Musaat, donde se acordó la realización de una investigación de ámbito nacional español sobre patologías en edificación.

\section{REFERENCIAS}

(1) Lee, D., Chi, H., Wang, J., Wang, X., Park, C. (2016). A linked data system framework for sharing construction defect information using ontologies and BIM environments. Automation in Construction, 68:102-113, doi: https://doi. org/10.1016/j.autcon.2016.05.003

(2) Neto, N., De Brito, J. (2011). Inspection and Defect Diagnosis System for Natural Stone Cladding. Journal of Materials in Civil Engineering, 23(10):1433-1443, doi: https://doi.org/10.1061/(ASCE)MT.1943-5533.0000314

(3) Gonçalves, A., De Brito, J., Amaro, B. (2014). Systematic approach to inspect, diagnose, and repair masonry walls. $J$ Perform ConstrFacil, 29(6):04014155, doi: https://doi.org/10.1061/(ASCE)CF.1943-5509.0000650

(4) Sá, G., Sá, J., De Brito, J., Amaro, .B. (2015). Statistical survey on inspection, diagnosis and repair of wall renderings. Journal of Civil Engineering and Management, 21(55):623-636, doi: https://doi.org/10.3846/13923730.2014.890666

(5) Garcez, N., Lopes, N., De Brito, J., Sá, G., Silvestre, J.D. (2015). Influence of design on the service life of pitched roofs' cladding. J Perform ConstrFacil, 29(3):04014073, doi: https://doi.org/10.1061/(ASCE)CF.1943-5509.0000461

(6) Gaspar, J., De Brito, J. (2011, 13 April). The Perception of Damage on Rendered Façades. 12th International Conference on Durability of Building Materials and Components (XII DBMC). Porto (Portugal).

(7) Mydin, A.O. (2015). Significance of building maintenance management system towards sustainable development: A review. Journal of Engineering Studies and Research, 21(1):58-65, doi: https://doi.org/10.29081/jesr.v21i1.41

(8) Chapman, J.C. (2001). Learning from failures. In: Campbell P. (Ed.), Learning from construction failures (Applied forensic engineering) (pp. 71-101). United Kingdom: Whittles Publishing.

(9) Carper, K.L. (2001). Forensic engineering: the perspective from North America. In: Campbell P. (Ed.), Learning from construction failures (Applied forensic engineering) (pp. 1-6). United Kingdom: Whittles Publishing.

(10) Marín, D., Moyano, J., Rico, F., Nieto, J. (2017). Modelo automatizado de tipificación de daños materiales por vicios o defectos que afecten a elementos de terminación y acabado en edificación. Informes de la Construcción, 69(545):182, doi: https://doi.org/10.3989/ic.15.105

(11) Ferguson, H., Clayton, L. (1988). Quality in the constructed project: a guideline for owners, designers and constructors. 1st ed. New York (USA): ASCE.

(12) Castellano, J., Ribera, A., Ciurana, J. (2016). Integrated system approach to evaluate social, environmental and economics impacts of buildings for users of housings. Energy Build, 123:106-118, doi: https://doi.org/10.1016/j. enbuild.2016.04.046 
(13) Mesa Fernandez, J.M., Palacios González, C., Álvarez Cabal, V., Villanueva Balsera, J. (2016). Analysis of the quality control planning in residential construction projects in Spain. Revista de la Construcción, 15(2), doi: https://doi. org/10.4067/So718-915X2016000200011

(14) Mills, A., Love, P.E., Williams, P. (2009). Defect Costs in Residential Construction. Journal of Construction Engineering and Management, 135(1):12-16, doi: https://doi.org/10.1061/(ASCE)0733-9364(2009)135:1(12)

(15) Mateus, R., Neiva, S., Bragança, L., Mendonça, P., Macieira, M. (2013). Sustainability assessment of an innovative lightweight building technology for partition walls-comparison with conventional technologies. Build Environ, 67:147-159, doi: https://doi.org/10.1016/j.buildenv.2013.05.012

(16) Taggart, M., Koskela, L., Rooke, J. (2014). The role of the supply chain in the elimination and reduction of construction rework and defects: an action research approach. Constr. Manage. Econ., 32(7-8):829-842, doi: https://doi.org/10.108 o/01446193.2014.904965

(17) Schiavoni, S., Bianchi, F., Asdrubali, F. (2016). Insulation materials for the building sector: A review and comparative analysis. Renewable and Sustainable Energy Reviews, 62:988-1011, doi: https://doi.org/10.1016/j.rser.2016.05.045

(18) Gaião, C., De Brito, J., Silvestre, J. (2010). Inspection and diagnosis of gypsum plasterboard walls. J Perform ConstrFacil, 25(3):172-180, doi: https://doi.org/10.1061/(ASCE)CF.1943-5509.0000149

(19) Delgado, A., Pereira, C., De Brito J., Silvestre, J. (2018) Defect characterization, diagnosis and repair of wood flooring based on a field survey. Materiales de Construcción, 68(329):149, doi: https://doi.org/10.3989/mc.2018.01817

(20) Galvão, J., Flores-Colen, I., De Brito, J., Veiga, M. (2018). Variability of in-situ testing on rendered walls in natural ageing conditions-Rebound hammer and ultrasound techniques. ConstrBuild Mater, 170:167-181, doi: https://doi. org/10.1016/j.conbuildmat.2018.02.152

(21) MUSAAT (2008-2010). Expedientes e informes periciales de siniestros. Madrid: Mutua de Aparejadores y Arquitectos Técnicos.

(22) Carretero-Ayuso, M.J., Moreno-Cansado, A. (2013). Análisis estadístico nacional sobre patologías en la edificación: años 2008 a 2010. Madrid: Fundación MUSAAT.

(23) SERJUTECA (2008-2010). Expedientes y documentación de siniestros de responsabilidad civil profesional de aparejadores y arquitectos técnicos. Madrid: Servicios Jurídicos Técnicos Aseguradores.

(24) INE (2017). Producto interior bruto de España. Renta nacional. Contabilidad nacional anual de España. Madrid: Instituto Nacional de Estadística.

(25) Munera, I. (2018). SOS: Se busca mano de obra especializada en la construcción. Madrid: Unidad Editorial.

(26) Karim, K., Marosszeky, M., Davis, S. (2006). Managing subcontractor supply chain for quality in construction. Engineering, Construction and Architectural Management, 13(1):27-42, doi: https://doi.org/10.1108/09699980610646485

(27) Villanueva, D. (1766). Colección de diferentes papeles críticos sobre todas las partes de la Arquitectura. Valencia: Imprenta Joachim Ibarra.

(28) Palladio, A. (1797). I quattro libri dell'architettura. [Reedición de la obra impresa por la Imprenta Real de Madrid, 1570]. Venecia.

(29) Forcada, N., Macarulla, M., Gangolells, M., Casals, M. (2016). Handover defects: comparison of construction and posthandover housing defects. Build. Res. Inf., 44(3):279-288, doi: https://doi.org/10.1080/o9613218.2015.1039284

(30) Spairani Berrio, S., García Alcocel, E.M., Roca Cladera, J. (2011). The impact of quality building materials on the satisfaction of the individual: an analysis based on subjective perception survey. Architecture, City and Environment, 5(15):7394, doi: https://doi.org/10.5821/ace.v5i15.2512 